\title{
How do we infer shell effects at high-excitation energies? A new spectroscopic probe to search for magic numbers
}

\author{
Cebo Ngwetsheni ${ }^{1, *}$ and José Nicolás Orce ${ }^{1, * *}$ \\ ${ }^{1}$ Department of Physics \& Astronomy, University of the Western Cape, P/B X17 Bellville ZA-7535, South Africa
}

\begin{abstract}
The nuclear dipole polarizability is mainly governed by the dynamics of the giant dipole resonance and, assuming validity of the brink-Axel hypothesis, has been investigated along with the effects of the lowenergy enhancement of the photon strength function for nuclides in medium- and heavy-mass nuclei. Cubicpolynomial fits to both data sets extrapolated down to a gamma-ray energy of $0.1 \mathrm{MeV}$ show a significant reduction of the nuclear dipole polarizability for semi-magic nuclei, with magic numbers $N=28,50$ and 82 , which supports shell effects at high-excitation energies in the the quasi-continuum region. This work assigns $\sigma_{-2}$ values as sensitive measures of long-range correlations of the nuclear force and provides a new spectroscopic probe to search for "old" and "new" magic numbers at high-excitation energies.
\end{abstract}

\section{Motivation}

Atomic masses are useful measures to study nuclear structure via nuclear binding energies. Deviations from the smooth trend presented by two-neutron separation energies can be associated with a sudden onset of deformation [1] or the rise of a magic number [2]. This information depends on high-precision measurements of atomic masses and is limited to ground and isomeric states of nuclei [3]. In this work, we propose an alternative spectroscopic probe, namely the nuclear polarizability, to investigate the rise of magic numbers at high-excitation energies. This work expands on the data analysis of our recent publications $[4,5]$ by doing a systematics study for the limited available information and assuming that the enhancement of the photon-strength function continues at lower gamma-ray energies of $E_{\gamma} \geqq 0.1 \mathrm{MeV}$. Overall, this cutoff shows a greater sensitivity of the nuclear polarizability to the long-range correlations in the nuclear wave functions, as realized from relative sharp drops of the nuclear polarizability in semi-magic nuclei.

The nuclear polarizability is dominated by the dynamics of the isovector giant dipole resonance (GDR) [6, 7], which is microscopically described as a coherent superposition of 1 particle - 1 hole (1p - 1h) excitations [8], and, macroscopically, as the inter-penetrating motion of proton and neutron fluids out of phase. The latter results from the nuclear symmetry energy, $a_{\text {sym }}$, in the Bethe-Weizsäcker semi-empirical mass formula $[9,10]$ acting as a restoring force [6], $a_{\text {sym }}\left(\rho_{N}-\rho_{Z}\right)^{2} / \rho_{A}$, where $\rho_{N}, \rho_{Z}$ and $\rho_{A}$ are the neutron, proton and total mass densities, respectively. Using the liquid drop model, with potential energy $\rho_{z}$, Migdal

\footnotetext{
*e-mail: 3344489@myuwc.ac.za

**e-mail: jnorce@uwc.ac.za
}

calculated the ground state (g.s.) electric dipole polarizability $\alpha_{E 1}=\frac{\mathbf{P}}{\mathbf{E}}$, where $\mathbf{P}$ is the electric dipole moment and $\mathbf{E}$ the electric field strength, connecting to $a_{\text {sym }}$ as follows,

$$
\alpha_{E 1}=\frac{e^{2} R^{2} A}{40 a_{\text {sym }}}=2.25 \times 10^{-3} A^{5 / 3} \mathrm{fm}^{3},
$$

where $a_{\text {sym }}=23 \mathrm{MeV}$ was assumed by Migdal as well as a defined spherical surface of radius $R=1.2 A^{1 / 3} \mathrm{fm}$ [6]. Hence, $\alpha_{E 1}$ is proportional to the size and diffuseness of the nucleus. As a second-order effect in perturbation theory, $\alpha_{E 1}$ is related to the total cross section $\sigma_{\text {total }}$ and its $(-2)$ moment, $\sigma_{-2}$, in the following manner [11],

$$
\alpha_{E 1}=2 e^{2} \sum_{n} \frac{\langle i\|\hat{E} 1\| n\rangle\langle n\|\hat{E} 1\| i\rangle}{E_{\gamma}}=\frac{\hbar c}{2 \pi^{2}} \sigma_{-2},
$$

where $\hat{E} 1$ is the electric dipole operator, $|i\rangle$ and $|n\rangle$ are the ground and excited state vectors and $\sigma_{-2}$ is defined as,

$$
\sigma_{-2}:=\int_{0}^{E_{\gamma_{\max }}} \frac{\sigma_{\text {total }}\left(E_{\gamma}\right)}{E_{\gamma}^{2}} d E_{\gamma}
$$

where $E_{\gamma_{\max }}$ is the maximum $E_{\gamma}$ available experimentally [12]. Combining Eqs. 1 and 2, Migdal determined,

$$
\sigma_{-2}=2.25 A^{5 / 3} \mu \mathrm{b} / \mathrm{MeV} \text {. }
$$

A new empirical formula for $\sigma_{-2}$ [13] has been determined from the 1988 photoneutron cross-section evaluation using monoenergetic photons [12], which agrees with Migdal's power-law formula,

$$
\sigma_{-2}=2.4 \kappa A^{5 / 3} \mu \mathrm{b} / \mathrm{MeV},
$$

where the polarizability parameter $\kappa$ is included to account for deviations from the actual GDR effects to that predicted 
by the hydrodynamic model $[6,14]$. The polarizability parameter $\kappa$ can therefore be extracted for known $\sigma_{-2}$ values and vice versa.

Generally, $\sigma_{-2}$ values should include both electric $\left(\alpha_{E 1}\right)$ and magnetic $\left(\chi_{M 1}\right)$ dipole polarizabilities [15],

$$
\sigma_{-2}=\frac{2 \pi^{2}}{\hbar c}\left(\alpha_{E 1}+\chi_{M 1}\right)
$$

Similarly, $\chi_{M 1}$ is a measure of the magnetic dipole response, $\mathbf{M}$, to a magnetic field, $\mathbf{B}$, i.e. $\mathbf{M}=\chi_{M 1} \cdot \mathbf{B}$ [16], and can be decomposed into paramagnetic $\left(\chi_{M 1}^{\text {para }}\right)$ and diamagnetic $\left(\chi_{M 1}^{d i a}\right)$ susceptibilities,

$$
\begin{aligned}
\chi_{M 1} & =\chi_{M 1}^{\text {para }}+\chi_{M 1}^{\text {dia }} \\
& =2 \sum_{n} \frac{\langle i\|\hat{M} 1\| n\rangle\langle n\|\hat{M} 1\| i\rangle}{E_{\gamma}}-\frac{Z e^{2}}{6 m c^{2}}\left\langle r^{2}\right\rangle .
\end{aligned}
$$

Permanent magnetic dipole moments, i.e. paramagnetism, is dominant for $A<20$ nuclides and may have a substantial contribution to $\sigma_{-2}$ values (e.g. ${ }^{6} \mathrm{Li}$ and ${ }^{7} \mathrm{Li}$ ), whereas diamagnetism has negligible effects $[15,16]$. It would be interesting to have an updated evaluation of $M 1$ strengths to investigate whether paramagnetism contributes in heavy nuclei, particularly from mixedsymmetry states recently found in the $A \sim 90$ region [1719].

Because of the $1 / E_{\gamma}{ }^{2}$ weighting in Eq. 3, $\sigma_{-2}$ values are not significantly affected by contributions above the GDR energy (e.g. recent discrepancies found between the Livermore and Saclay photoneutron data with monochromatic photon beams [20, 21] or nucleon resonances at $E_{\gamma} \gtrsim 140 \mathrm{MeV}$ [22]), and are extremely sensitive measures - unlike $\sigma_{\text {total }}$ - of long-range correlations in the nuclear wave functions.

\section{Low-energy enhancement of the photon strength function}

The photon strength function $f\left(E_{\gamma}\right)$ characterizes average EM decay and absorption properties of excited nuclei. Recent measurements of $f\left(E_{\gamma}\right)$ by the Oslo group have revealed an enhancement at low $E_{\gamma}$ [23-26]. These measurements are performed in the quasi-continuum energy region and assume the validity of the Brink-Axel hypothesis $[27,28]$, which states that $f\left(E_{\gamma}\right)$ is independent of the particular structure of the initial and final state [29] and only depends on $\mathrm{E}_{\gamma}$, i.e. GDR properties are similar for all nuclear states. To date, the EM character of the LEE remains undetermined experimentally, although polarization asymmetry measurements of $\gamma$ rays in ${ }^{56} \mathrm{Fe}$ show an admixture of $M 1$ and $E 1$ radiation [30]. Various interpretations of the LEE have been proposed, explaining its dipole origin as $M 1$ [31-36] and $E 1$ [37] dipole radiation [38]. Shell-model (SM) calculations consistently support the $M 1$ nature of the LEE [31-34]. The main purpose of this work is to quantify the potentially large contribution from the LEE to the nuclear polarizability and $\sigma_{-2}$ values assuming dipole radiation and validity of the Brink-Axel hypothesis.

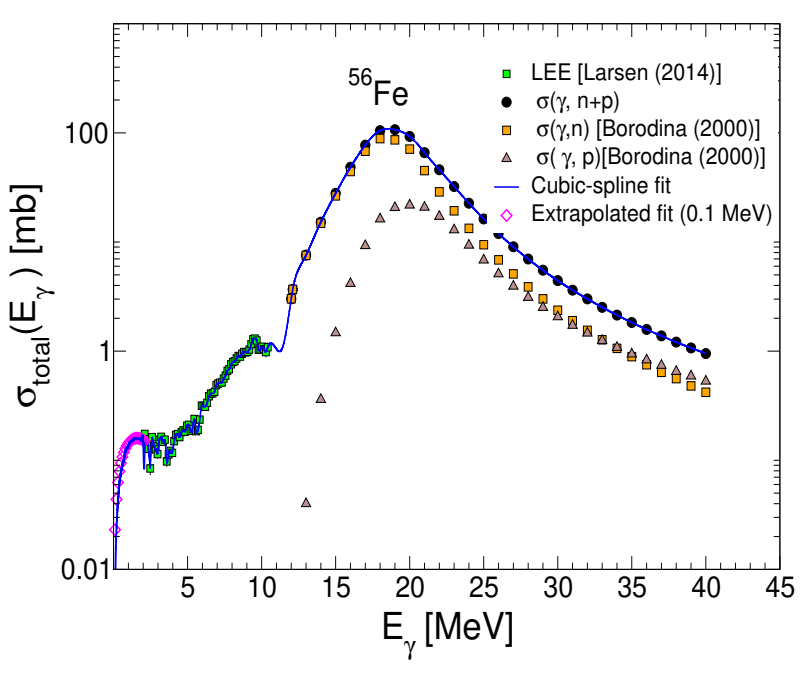

Figure 1. $\sigma_{\text {total }}\left(E_{\gamma}\right)$ vs $E_{\gamma}$ on a $\log$ scale on the y-axis showing the interpolation function data (solid blue line) for ${ }^{56} \mathrm{Fe}$, the green squares represent $\sigma_{L E E}\left(E_{\gamma}\right)$ and black filled circles the sum of proton and neutron emission cross sections for the GDR.

\section{Systematics of total $\sigma_{-2}$ values and effect in the nuclear polarizability}

The LEE is generally observed in medium-mass nuclei in the $A \approx 50$ and 90 mass regions and only for ${ }^{105} \mathrm{Cd}$, ${ }^{138,139} \mathrm{La}$ and ${ }^{151,153} \mathrm{Sm}$ [39-41] in heavy-mass nuclei. These nuclei, spanning the mass range $A=45-153$, have been considered in order to obtain a systematic study of LEE + GDR effects on $\sigma_{-2}$ values, which requires the combined analysis of LEE and GDR cross sections,

$$
\sigma_{\text {total }}\left(E_{\gamma}\right)=\sigma_{G D R}\left(E_{\gamma}\right)+\sigma_{L E E}\left(E_{\gamma}\right),
$$

where $\sigma_{G D R}\left(E_{\gamma}\right)=\sigma(\gamma, p)+\sigma(\gamma, n)+\sigma(\gamma, 2 n)+\sigma(\gamma, n p)$ is given by photo-nuclear absorption reactions at energies above nucleon threshold, and $\sigma_{L E E}\left(E_{\gamma}\right)$ is the cross section contribution from the LEE region below the neutron threshold. The GDR data are obtained from the experimental nuclear-reaction databases EXFOR [42] and ENDF [43], whereas the LEE data come from the Oslo compilation of $f\left(E_{\gamma}\right)$ [44], in units of $\mathrm{MeV}^{-3}$. The LEE data can be converted to cross sections as follows [45],

$$
\sigma_{L E E}\left(E_{\gamma}\right)=\pi^{2} g_{J}(\hbar c)^{2} f\left(E_{\gamma}\right) E_{\gamma}[\mathrm{mb}],
$$

where $g_{J}$ is the statistical factor $g_{J}=\frac{2 J_{J}+1}{2 J_{i}+1}$ with spins $J_{i}$ and $J_{f}$ corresponding to initial and final states, respectively. The magnitude of $g_{J}$ affects $\sigma_{-2}$ and polarizability values proportionally. Considering the dipole character of the LEE, an average $\left\langle g_{J}\right\rangle=1$ is a reasonable approximation for dipole $\Delta J=0$ and $\Delta J=1$ transitions.

The combination of GDR and LEE contributions may be arguable a priori, because $\sigma_{G D R}\left(E_{\gamma}\right)$ corresponds to transitions between excited states $|n\rangle$ in the GDR region and the g.s. $|i\rangle$, whereas $\sigma_{L E E}\left(E_{\gamma}\right)$ results from transitions between excited states in the quasi-continuum region. Recent studies of $f\left(E_{\gamma}\right)$ by Guttormsen and co-workers 

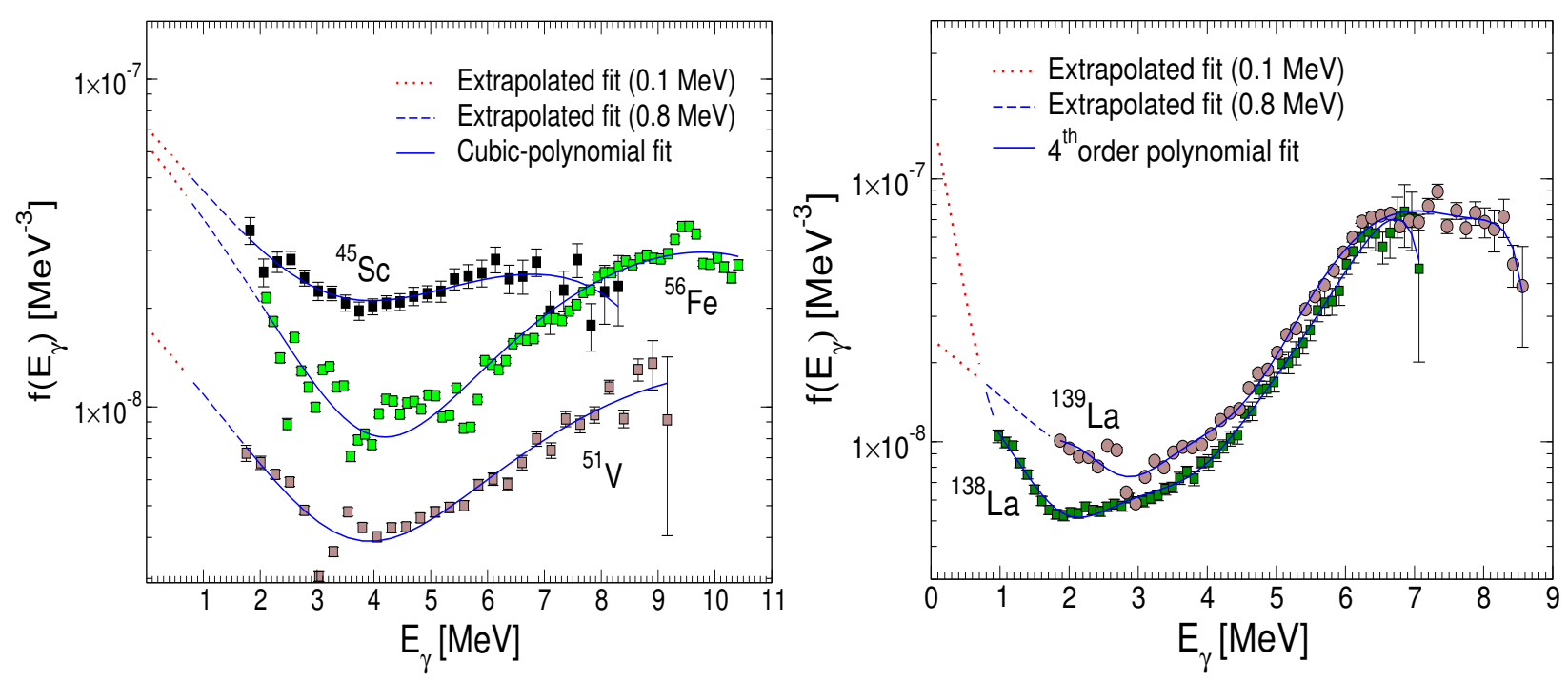

Figure 2. $f\left(E_{\gamma}\right)$ for ${ }^{45} \mathrm{Sc},{ }^{51} \mathrm{~V}$ and ${ }^{56} \mathrm{Fe}$ (left panel) fitted with a cubic polynomial and ${ }^{138,139} \mathrm{La}$ (right panel) fitted with a $4^{\text {th }}$-order polynomial, including extrapolated data to $E_{\gamma_{\min }}=0.8 \mathrm{MeV}$ (blue dashed line) and $E_{\gamma_{\min }}=0.1 \mathrm{MeV}$ (red dotted line).

support the validity of the Brink-Axel hypothesis at different bin energies in the quasi-continuum region [46]. This, together with the fact that GDR studies of nuclei at relatively low temperatures $T$ and spins $J$ and cold nuclei in their ground state present similar features [47, 48], may allow for combining the LEE and GDR cross sections [4]. As shown in Figs. 1 and 2, it is somewhat surprising that the data from the low-energy tail of the GDR generally merge nicely with the LEE data without applying any normalization factor. Nonetheless, the validity for the combination of these data needs further experimental confirmation.

An interpolation method for calculating $\sigma_{\text {total }}\left(E_{\gamma}\right)$ and $\sigma_{-2}$ values has been used in this work. This method is independent of any physical phenomena and operates by creating a function - cubic or $4^{\text {th }}$ order polynomial - that interpolates (add data) between the fixed experimental data points. As an example, Fig. 1 shows the total cross section of ${ }^{56} \mathrm{Fe}$ with a cubic-spline interpolation function (solid blue line). The resulting functions are integrated accordingly to obtain $\sigma_{\text {total }}\left(E_{\gamma}\right)$, which yields the $\sigma_{-2}$ values listed in Table 1. Most nuclei present an energy gap with missing data between the LEE and GDR data, which may include the $M 1$ spin-flip resonance and the pygmy dipole resonances (PDR) in neutron-rich nuclei. Therefore, data from ENDF [43] - when available have been used to fill the gap [4, 5]. Additionally, data near nucleon threshold energies generally present large uncertainties and have been excluded.

Because of minimal RMS errors, a cubic-polynomial interpolation has been selected as the interpolating function throughout this work. Similar results are often obtained using a $4^{\text {th }}$-order polynomial interpolation. Lower and higher order polynomial interpolations present unexpected structures (bumps) in the energy-gap region and above. The errors associated to $\sigma_{-2}$ values are calculated from upper and lower loci limits of $\sigma_{\text {total }}\left(E_{\gamma}\right)$, including LEE and GDR contributions, which yield an uncertainty of $7 \%$. Uncertainties for the interpolation data spanning the gap and extrapolated data are treated as three points standard deviation of the mean, in order to determine the upper and lower limits for these data sets. Most of the considered nuclei are stable, except ${ }^{50} \mathrm{~V},{ }^{138} \mathrm{La}$ and ${ }^{153} \mathrm{Sm}$ with no experimental GDR cross sections. Therefore, GDR data of stable neighboring isotopes were used, i.e. ${ }^{51} \mathrm{~V},{ }^{139} \mathrm{La}$ and ${ }^{152} \mathrm{Sm}$, under the assumption that neighboring nuclei present similar $f\left(E_{\gamma}\right)[65]$ and considering the fact that $\sigma_{-2}$ values show a strong dependence on nuclear mass $A$.

Setting up the low-energy cut-off, $E_{\gamma_{\min }}$, for the LEE is not obvious. In our previous study, we extrapolated the LEE data down to $800 \mathrm{keV}$ from experimental observations which show $E_{\gamma_{\min }} \approx 1 \mathrm{MeV}$ for most nuclei, except for ${ }^{153} \mathrm{Sm}$ where measurements were carried out down to $E_{\gamma_{\min }}=645 \mathrm{keV}$. Recent SM studies [30, 33, 35] explore, however, the behavior of $f\left(E_{\gamma}\right)$ at very low $E_{\gamma}$, supporting the continuation of the LEE down to $E_{\gamma_{\min }}=0$. We have investigated this situation extrapolating the data to $E_{\gamma_{\min }}=0.1 \mathrm{MeV}$. It is not obvious to us the physical meaning of going down to $E_{\gamma_{\min }}=0$ without any further experimental evidence. Consequently, Fig. 2 shows extrapolated fits of $f\left(E_{\gamma}\right)$ data down to $E_{\gamma_{\min }}=0.1 \mathrm{MeV}$. Similar fits were done for all the considered nuclei to explore the evolution of $f\left(E_{\gamma}\right)$ towards $E_{\gamma_{\max }} \approx 0$. The results are listed in Table 1.

A large enhancement of $\sigma_{-2}$ values is found for ${ }^{45} \mathrm{Sc}$ and ${ }^{56} \mathrm{Fe}$ and ${ }^{153} \mathrm{Sm}$ as compared with semi-magic nuclei ${ }^{51} \mathrm{~V}$ and ${ }^{139} \mathrm{La}$. If these predictions of $f\left(E_{\gamma}\right)$ for $E_{\gamma_{\min }} \rightarrow 0$ $\mathrm{MeV}$ are consistent with experimental findings, reaction rates in nucleosynthesis following rapid-neutron capture the $r$-process - may strongly be affected together with the predicted abundances of nuclei [40, 69-71]. Additional fits using Eq. 4 in Ref. [69] have been performed, which 


\begin{tabular}{lccccccc}
\hline \hline Nucleus & $\begin{array}{c}\mathrm{E}_{\gamma(\min )} \\
(\mathrm{MeV})\end{array}$ & $\begin{array}{c}\mathrm{E}_{\gamma(\max )}(\mathrm{GDR}) \\
(\mathrm{MeV})\end{array}$ & $\begin{array}{c}\mathrm{E}_{\gamma(\max )}(\mathrm{LEE}) \\
(\mathrm{MeV})\end{array}$ & $\begin{array}{c}\sigma_{-2}(\text { total }) \\
(\mu \mathrm{b} / \mathrm{MeV})\end{array}$ & $\begin{array}{c}\sigma_{-2}(\mathrm{LEE}) \\
(\mu \mathrm{b} / \mathrm{MeV})\end{array}$ & $\begin{array}{c}\text { C } \\
\%\end{array}$ & $\kappa$ \\
\hline${ }^{45} \mathrm{Sc}^{*}$ & 0.1 & 28.1 & 3.2 & $2488(602)$ & 873 & $35.1 \%$ & $1.82(4)$ \\
${ }_{50} \mathrm{~V}$ & 0.1 & 27.8 & 3.1 & $1597(105)$ & 187 & $11.7 \%$ & $0.98(1)$ \\
${ }_{53}^{21} \mathrm{~V}$ & 0.1 & 27.8 & 3.1 & $1564(273)$ & 140 & $9.1 \%$ & $0.92(2)$ \\
${ }^{23} \mathrm{~V}$ & 0.1 & 40.0 & 3.8 & $2658(354)$ & 568 & $21.4 \%$ & $1.35(2)$ \\
${ }^{56} \mathrm{Fe}^{*}$ & 0.1 & 26.5 & 2.3 & $3291(1279)$ & 135 & $4.1 \%$ & $1.00(4)$ \\
${ }_{32} \mathrm{Ge}$ & 0.1 & 27.8 & 2.2 & $3166(528)$ & 199 & $6.3 \%$ & $0.70(1)$ \\
${ }_{92} \mathrm{Zr}$ & 0.1 & 27.8 & 2.5 & $5017(673)$ & 340 & $6.8 \%$ & $1.05(1)$ \\
${ }_{95} \mathrm{Mo}$ & 0.1 & 1.9 & $8580(1240)$ & 319 & $7.3 \%$ & $0.97(1)$ \\
${ }_{13}^{13} \mathrm{La}$ & 0.1 & 24.3 & 2.5 & $8183(837)$ & 221 & $2.7 \%$ & $0.91(1)$ \\
${ }_{57}^{139} \mathrm{La}$ & 0.1 & 24.3 & 1.6 & $12998(1638)$ & 3093 & $23.9 \%$ & $1.23(2)$ \\
${ }_{57}^{53} \mathrm{Sm}$ & 0.1 & 20.0 & & & & & \\
62
\end{tabular}

Table 1. Contributions of the LEE with $E_{\gamma_{\min }}=0.1 \mathrm{MeV}$ represented by $\mathbf{C}$. $\mathrm{E}_{\gamma(\max )}(\mathrm{LEE})$ is the energy at which the LEE starts.

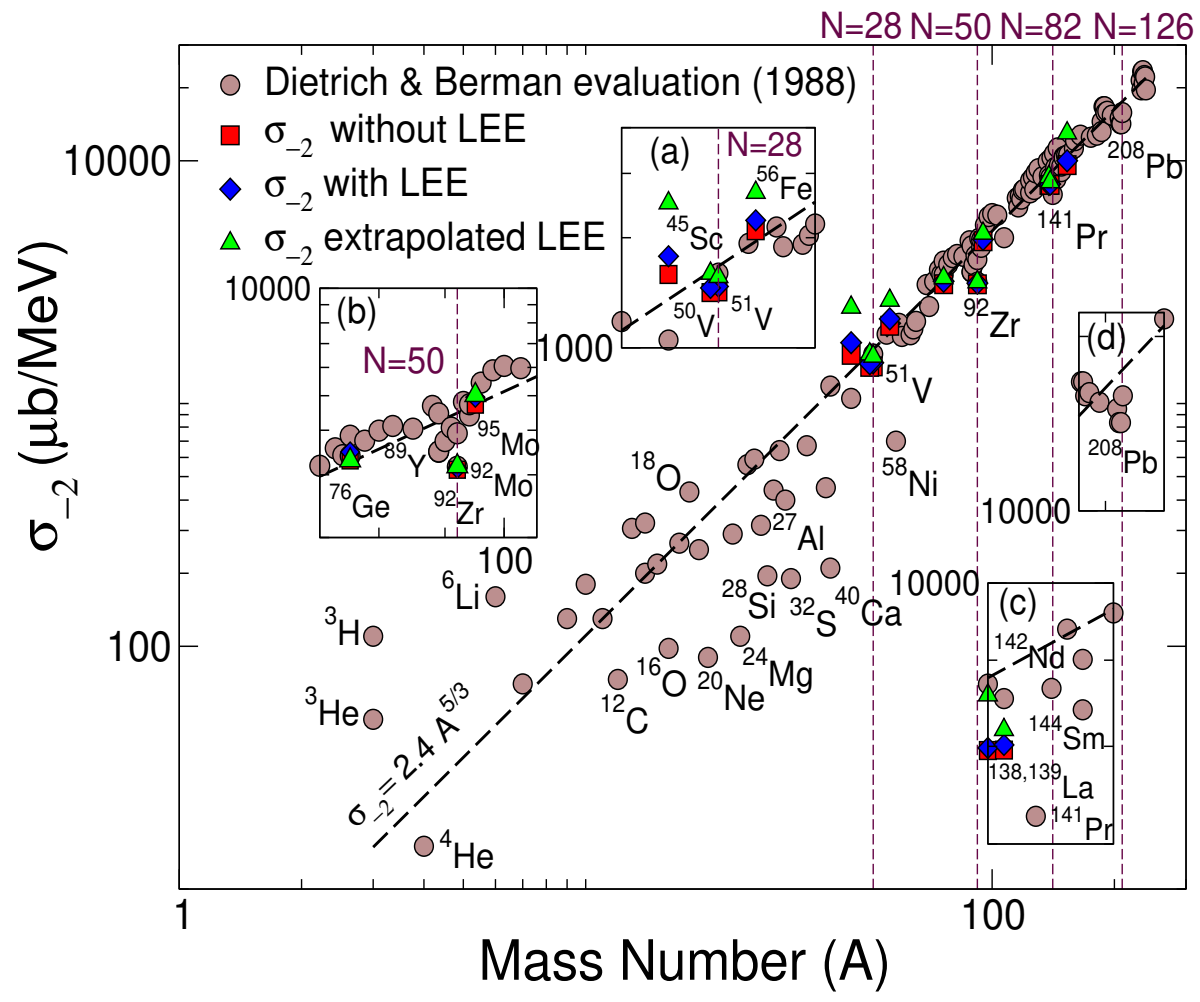

Figure 3. $\sigma_{-2}$ vs A on a log-log scale from the photoneutron cross-section evaluation (solid circles) [12] and $\sigma_{-2}$ data listed in Table 1 excluding (squares) and including (diamonds) the LEE contributions down to $E_{\gamma}=0.1 \mathrm{MeV}$. For comparison, the empirical formula $\sigma_{-2}=2.4(1) A^{5 / 3} \mu \mathrm{b} / \mathrm{MeV}$ (dashed line) [13] is plotted. Shell effects are noticeable for $N=28$ (inset (a)), $N=50$ (inset (b)), 82 (inset (c)) and 126 (inset (d)) isotones.

show an even larger LEE contribution, but no indications of shell effects when comparing neighboring nuclei.

\section{Discussion}

Shell effects arising from $\sigma_{-2}$ values are evident in nuclei with or near magic numbers $N=28,50,82$ and 126. As shown in Fig. 3, a more distinct trend of $\sigma_{-2}$ values emerges when the LEE contributions are extrapolated to $E_{\gamma_{\min }}=0.1 \mathrm{MeV}$, with a relatively smaller contribution to $\sigma_{-2}$ values in ${ }^{51} \mathrm{~V}(N=28),{ }^{92} \mathrm{Zr}(N=50$ and $Z=40)$ and ${ }^{139} \mathrm{La}(N=82)$, as compared with neighboring nuclides. As listed in Table 1, these semimagic nuclei present smaller polarizability parameters of $\kappa \approx 0.90$ for ${ }^{51} \mathrm{~V}$ and ${ }^{139} \mathrm{La}$ and an even smaller $\kappa=0.70$ for ${ }^{92} \mathrm{Zr}$. These findings, assuming validity of the BrinkAxel hypothesis, support the continuation of shell effects in the quasi-continuum region where the LEE of $f\left(E_{\gamma}\right)$ occurs. The fact that the nuclear polarizability can change at higher excitation energies from its ground-state value is supported by different $\kappa$ values predicted by consistent nocore-shell-model calculations in ${ }^{12} \mathrm{C}$ - with a larger $\kappa$ value determined for the first $2^{+}$excitation at $4.439 \mathrm{MeV}$ with respect to the ground state - using both $2 N$ and $2 N+3 N$ chiral forces [72]. 
Furthermore, Table 1 shows considerable LEE contributions to $\sigma_{-2}$ values for more deformed nuclei such as ${ }^{45} \mathrm{Sc},{ }^{56} \mathrm{Fe}$ and ${ }^{153} \mathrm{Sm}$ with $\kappa>1$ values, while other nuclei close to magic numbers present consistent values of $\kappa \approx 1$. Certainly from these results, $E_{\gamma_{\min }}$ is crucial for determining how the LEE affects the nuclear polarizability. Additionally, the LEE contribution is affected by the smaller $E_{\gamma_{\max }}(\mathrm{LEE})$ for heavy nuclei and the inverse mass dependence of $\mathrm{E}_{G D R}[7,68]$.

The sudden drop of $\sigma_{-2}$ values and $\kappa<1$ for nuclei with neutron magic numbers $N=28,50$ and 82 supports the $M 1$ nature of the LEE predicted by large-scale SM calculations and the validity of the generalized Brink-Axel hypothesis, which seems to allow for structural changes. This work emphasizes the need for additional $f\left(E_{\gamma}\right)$ measurements at low energies below $1 \mathrm{MeV}$, and opens a new research avenue to investigate the existence and evolution of magic numbers at high-excitation energies from $\sigma_{-2}$ measurements [4].

Finally, it is relevant to note that the combination of data sets with such a distinct nature in this study, namely LEE and GDR data, remains unsettling and needs to be explored further; for instance, the Brink-Axel hypothesis could additionally be supported with the presence of LEE associated with GDRs built on excited states, preferably around the quasi-continuum region. More experimental $f\left(E_{\gamma}\right)$ measurements and theoretical work are clearly needed to validate these interesting findings.

\section{Acknowledgements}

This work was supported by the National Research Foundation of South Africa under Grant 93500, the MANuS/MatSci program and the SA-CERN collaboration.

\section{References}

[1] U. Hager et al., Phys. Rev. Lett. 96, 069901 (2006).

[2] F. Wienholtz et al., Nature 498, 346 (2013).

[3] J. Dilling, K. Blaum, M. Brodeur, and S. Eliseev, Annu. Rev. Nucl. Part. Sci. 68, 45 (2018).

[4] C. Ngwetsheni and J. N. Orce, Phys. Lett. B 792, 335 (2019).

[5] C. Ngwetsheni and J. N. Orce, to be published in Hyperfine Interactions (2019).

[6] A. Migdal, J. Exptl. Theoret. Phys. U.S.S.R. 15, 81 (1945).

[7] H. Steinwedel, J. H. D. Jensen, and P. Jensen, Phys. Rev. 79, 1019 (1950).

[8] M. Danos, B. S. Ishkhanov, N. P. Yudin, and R. A. Eramzhyan, Phys. Uspekhi 37(12), 1297 (1995).

[9] H. A. Bethe and R. F. Bacher, Rev. Mod. Phys. 8, 82 (1936).

[10] C. F. von Weizsäcker, Z. Phys. 96, 431 (1935).

[11] A. B. Migdal, A. A. Lushnikov, and D. F. Zaretsky, Nucl. Phys. A 66, 193 (1965).

[12] S. S. Dietrich and B. L. Berman, Atom. Data Nucl. Data Tables 38, 199 (1988).
[13] J. N. Orce, Phys. Rev. C 91, 064602 (2015)

[14] J. S. Levinger, Phys. Rev. 107, 554 (1957).

[15] W. Knüpfer and A. Richter, Phys. Lett. B 107, 325 (1981).

[16] W. Knüpfer and A. Richter, Z. Phys. A 320, 253 (1985).

[17] N. Pietralla et al., Phys. Rev. Lett. 83, 1303 (1999).

[18] C. Fransen et al., Phys. Rev. C 67, 024307 (2003).

[19] J. N. Orce et al., Phys. Rev. Lett. 97, 062504 (2006).

[20] B. S. Ishkhanov et al., Phys. At. Nucl. 67, 1664 (2004).

[21] I. Gheorghe et al., Phys. Rev. C 96, 044604 (2017).

[22] J. Ahrens, H. Gimm, A. Zieger and B. Ziegler, Il Nuovo Cimento A 32, N. 3, 364 (1976).

[23] A. Voinov et al., Phys. Rev. Lett. 93, 142504 (2004).

[24] A. Schiller et al., Nucl. Instr. Meth. Phys. Res. A 447, 498 (2000).

[25] M. Guttormsen et al., Nucl. Instr. Meth. Phys. Res. A 255, 518 (1987).

[26] M. Wiedeking et al., Phys. Rev. Lett. 108, 162503 (2012).

[27] D. Brink, doctoral thesis, Oxford University, 1955 (unpublished).

[28] P. Axel, Phys. Rev. 126, 671 (1962).

[29] D. Martin et al., Phys. Rev. Lett. 119, 182503 (2017).

[30] M. D. Jones et al., Phys. Rev. C 97, 024327 (2018).

[31] R. Schwengner, S. Frauendorf and A. C. Larsen, Phys. Rev. Lett. 111, 232504 (2013).

[32] B. A. Brown and A. C. Larsen, Phys. Rev. Lett. 113, 252502 (2014).

[33] K. Sieja, Phys. Rev. Lett. 119, 052502 (2017).

[34] K. Sieja, EPJ Web of Conferences 146, 05004 (2017).

[35] J.E. Midtbo et al., Phys. Rev. C 98, 064321 (2018).

[36] A. C. Larsen et al., Phys. Rev. C 97, 054329 (2018).

[37] E. Litvinova and N. Belov, Phys. Rev. C 88, 031302(R) (2013).

[38] J. D. Jackson, Classical Electrodynamics, John Wiley \& Sons, Inc. (1962).

[39] A. C. Larsen et al., Phys. Rev. C 87, 014319 (2013).

[40] B. V. Kheswa et al., Phys. Lett. B 744, 268 (2015).

[41] A. Simon et al., Phys. Rev. C 93, 034303 (2016).

[42] https://www-nds.iaea.org/exfor/exfor.htm

[43] https://www.nndc.bnl.gov/ensdf/

[44] http://www.mn.uio.no/fysikk/english/research/ about/infrastructure/OCL/nuclear-physicsresearch/compilation/

[45] M. Baranger, E. Vogt, Adv. in Nucl. Phys. 7, 229 (2012).

[46] M. Guttormsen et al., Phys. Rev. Lett. 116, 012502 (2016).

[47] A. Schiller and M. Thoennessen, Atom. Data Nucl. Data Tables 93, 549 (2007).

[48] J. J. Gaardhøje, Annu. Rev. Nucl. Part. Sci. 42, 483 (1992). 
[49] A. Vessiere et al., Nucl. Phys. A 227, 513 (1974).

[50] A. C. Larsen et al., Phys. Rev. C 76, 044303 (2007).

[51] K. Shoda, Nucl. Phys. A 239, 397 (1975).

[52] S. C. Fultz et al., Phys. Rev 128, 2345 (1962).,

[53] A. C. Larsen et al., Phys. Rev. C 73, 064301 (2006).

[54] S. S. Borodina et al., Moscow State Univ. Inst. of Nucl. Phys. Rep. 6 (2000).

[55] A. C. Larsen et al., Phys. Rev. Lett. 111, 242504 (2013).

[56] P. Carlos et al., Nucl. Phys. A 258, 365 (1976).

[57] A. Spyrou et al., Phys. Rev. Lett. 113, 232502 (2014).

[58] B. L. Berman et al., Phys. Rev. 162, 1098 (1967).

[59] M. Guttormsen et al., Phys. Rev. C 96, 024313 (2017).

[60] H. Utsunomiya et al., Phys. Rev. Lett. 100, 162502 (2008).

[61] H. Beil et al., Nucl. Phys. A 227, 427 (1974).
[62] H. Utsunomiya et al., Phys. Rev. C 88, 015805 (2013).

[63] H. Beil et al., Nucl. Phys. A 172, 426 (1971).

[64] P. Carlos et al., Nucl. Phys. A 225, 171 (1974).

[65] M. Guttormsen et al., Phys. Rev. C 71, 044307 (2005)

[66] B. L. Berman and S. C. Fultz, Rev. Mod. Phys. 47, 713 (1975).

[67] J. Tian, H. Cui, K. Zheng, and N. Wang, Phys. Rev. C 90, 024313 (2014).

[68] O. Bohigas, N. van Giai, and D. Vautherin, Phys. Lett. B 102, 105 (1981).

[69] A. C. Larsen and S. Goriely, Phys. Rev. C 82, 014318 (2010).

[70] M. Arnould, S. Goriely and K. Takahashi, Phys. Rep. 450, 97 (2007).

[71] A. C. Larsen, A. Spyrou, S. N. Liddick and M. Guttormsen, Prog. Part. Nucl. Phys. 107, 69 (2019).

[72] M. K. Raju et al., Phys. Lett. B. 777, 250 (2018). 